\title{
"Just think positive": We can all work to address the publication bias issue
}

Letter to the editor

377 words excluding references

David Berle

1. Discipline of Clinical Psychology, Graduate School of Health, University of Technology Sydney, Australia.

2. School of Psychiatry, UNSW Australia.

Address for correspondence:

David Berle

Discipline of Clinical Psychology

Graduate School of Health

Vicki Sara Building

University of Technology Sydney

67 Thomas Street

Ultimo NSW 2007

Australia

Ph: +61 295149221

Email: david.berle@uts.edu.au

Keywords: Publication, negative findings, methodology, research protocol. 
To the editor,

The recent editorial by Porter et al. (in press) raises important questions for psychiatric research. As the authors highlighted, distortions in the published literature reflect a waste of resources and increase the risk of clinical harm.

The preponderance of positive findings is not simply a consequence of editorial favoritism for positive and "newsworthy" results. Publication bias also exists at the level of individual researchers and the decisions we make about what we submit for publication and how we conduct and report our studies. These individual biases contribute to the publication landscape in a number of different disciplines.

In psychology, much of the discussion of these issues has been focused at the level of the individual researcher. When conducting a study, a researcher must make a series of seemingly innocuous decisions about when to stop recruitment, which observations to exclude, which moderating variables to consider and the most appropriate way of analyzing the results. These "researcher degrees of freedom" (Simmons et al., 2011) are thought to have a cumulative effect in tilting the likelihood of study findings towards the positive.

Pre-registration of research studies, as advocated by Porter et al., may help to restrain researchers from indulging in many of these potentially distorting "freedoms". However, pre-registration may not be sufficient in its own right (Goldacre, 2015). A further step would be to encourage researchers to submit their proposed research methodologies to peer review before commencing a study, as already occurs when researchers publish study protocols for clinical trials. This provides peer-reviewed legitimacy to one's research, and protects against negative findings being dismissed on purely methodological grounds. Researchers would then feel emboldened to submit their methodologically robust research for publication even when the findings are negative. When combined with other initiatives, such as efforts to ensure the open accessibility of data, these approaches hold promise for improving the integrity of the published literature.

There are of course increased administrative demands on researchers in attending to all these recommendations. On the other hand, there are also important potential benefits. Peer review of study protocols offers researchers increased recognition for their work, such that careers may become defined as much by the development and application of rigorous methodologies as by the chance significance or newsworthiness of one's results.

\section{Declaration of conflicting interests}

The author declared no potential conflict of interest with respect to the research, authorship and/or publication of this article.

\section{Funding}

This research received no specific grant from any funding agency in the public, commercial, or not-forprofit sectors. 


\section{References}

Goldacre B (2016) Make journals report clinical trials properly. Nature 530(7588): 7. DOI:

$10.1038 / 530007 a$

Porter RJ, Boden JM, Miskowiak K and Mahli GS (in press) Failure to publish negative results: A systematic bias in psychiatric literature. Australian and New Zealand Journal of Psychiatry. DOI: $10.1177 / 0004867416683816$

Simmons JP, Nelson LD and Simonsohn U (2011) False-positive psychology: Undisclosed flexibility in data collection and analysis allows presenting anything as significant. Psychological Science 22(11): 13591366. DOI: $10.1177 / 0956797611417632$ 